\title{
Interaction-Induced Quantum Dephasing in Mesoscopic Rings
}

\author{
Dmitri S. Golubev ${ }^{1,4}$, Carlos P. Herrero ${ }^{2}$ and Andrei D. Zaikin ${ }^{3,4}$ \\ ${ }^{1}$ Institut für Theoretische Festkörperphysik, Universität Karlsruhe, 76128 Karlsruhe, Germany \\ ${ }^{2}$ Instituto de Ciencia de Materiales de Madrid, CSIC, Cantoblanco, E-28049 Madrid, Spain \\ ${ }^{3}$ Forschungsentrum Karlsruhe, Institut für Nanotechnologie, 76021 Karlsruhe, Germany \\ ${ }^{4}$ I.E.Tamm Department of Theoretical Physics, P.N.Lebedev Physics Institute, 119991 Moscow, Russia
}

\begin{abstract}
Combining nonperturbative techniques with Monte Carlo simulations we demonstrate that quantum coherence effects for a particle on a ring are suppressed beyond a finite length $L_{\varphi}$ even at zero temperature if the particle is coupled to a diffusive electron gas by means of long range Coulomb interaction. This length is consistent with $L_{\varphi}$ derived from weak-localization-type of analysis.
\end{abstract}

Experiments [1] strongly suggest that decoherence time $\tau_{\varphi}$ of electrons in disordered conductors saturates to a finite value in the low temperature limit. Theoretical arguments [2] attribute this saturation to the effect of electron-electron interactions. This work has initiated intensive theoretical debates, the present status of which is summarized in recent publications [3].

Although detailed calculations of $\tau_{\varphi}$ for metallic conductors with disorder and interactions can be rather involved, the main cause for electron dephasing is quite clear without going into unnecessary complications: It is the electron interaction with the fluctuating quantum electromagnetic field produced by other electrons. A question of fundamental importance here is to understand whether such interaction can dephase at $T \rightarrow 0$, i.e. if the whole interacting system is in its true quantum mechanical ground state.

It is obvious that for a system in thermodynamic equilibrium quantum dephasing cannot be associated with any kind of decay or relaxation in real time, rather it manifests itself via suppression of off-diagonal elements of the electron density matrix beyond a certain length $L_{\varphi}$. Provided there exists nonzero electron dephasing due to its interaction with quantum environment even at $T \rightarrow 0$, this dephasing length $L_{\varphi}$ should stay finite down to zero temperature. This, in turn, would imply that effects sensitive to quantum coherence, such as, e.g., persistent currents $(\mathrm{PC})$ and Aharonov-Bohm (AB) oscillations in mesoscopic rings, should be suppressed by interactions at any temperature including $T=0$ if the perimeter of the ring exceeds $L_{\varphi}$.

Recently the authors 洎 demonstrated that the amplitude of PC can be reduced by interactions even exactly in the ground state, and this result was interpreted as a signature of suppression of quantum coherence at $T=0$. Other authors 5 argued that this reduction of $\mathrm{PC}$ is merely a renormalization effect which has nothing to do with dephasing. Very recently Guinea [6] found that $\mathrm{AB}$ oscillations for a quantum particle on a ring interacting with Caldeira-Leggett bath of oscillators are exponentially reduced beyond some length $L_{\varphi}$ which is set by interactions and remains finite down to zero temperature. Similar result was also obtained earlier from a real-time quasiclassical analysis [7]. It should also be noted that the model [6,7] is exactly mapped onto that of the so-called single electron box, the effective charging energy of which is well known to reduce exponentially at large conductances 8 11]. This reduction immediately translates into a finite dephasing length $L_{\varphi}$ at zero temperature found in 6.7. On the other hand, no finite $L_{\varphi}$ at $T=0$ was found in some other models [6, 12].

The purpose of this Letter is to investigate quantum dephasing for a model with long range Coulomb interactions in the presence of disorder. Our main conclusion is that such interactions can strongly suppress $\mathrm{PC}$ even in thermodynamic equilibrium at $T=0$.

The model and effective action. We will consider a quantum particle with mass $M$ on a ring with radius $R$ threaded by external magnetic flux $\Phi_{x}$. As in ref. [6] it will be convenient to describe the particle position by a vector $\boldsymbol{R}=(R \cos \theta, R \sin \theta)$ and consider the angle $\theta$ as a quantum variable. We also assume that the ring is embedded into an effective dissipative environment and the whole system is in equilibrium at a temperature $T$.

Our first and standard step is to integrate out the environmental degrees of freedom. After that the grand partition function of the system takes the form

$$
\mathcal{Z}=\sum_{m=-\infty}^{\infty} \int_{0}^{2 \pi m} \mathcal{D} \theta \exp \left(i 2 \pi m \phi_{x}-S_{0}[\theta]-S_{\text {int }}[\theta]\right) .
$$

The first term in the exponent takes care of the magnetic flux while the second term

$$
S_{0}[\theta]=\int_{0}^{\beta} d \tau \frac{1}{4 E_{C}}\left(\frac{\partial \theta}{\partial \tau}\right)^{2}
$$

is the particle action. Here we defined $\beta=1 / T, E_{C}=$ $1 /\left(2 M R^{2}\right)$ and $\phi_{x}=\Phi_{x} / \Phi_{0}$, where $\Phi_{0}$ is the flux quantum. The term $S_{\text {int }}$ describes the effect of interaction between the particle and the environment and, hence, depends both on the environment Hamiltonian and on the form of the interaction. We will assume that the particle has the electron charge $e$ which interacts with 
the fluctuating electromagnetic field $V$ produced by the effective environment. For this model one finds

$$
S_{\mathrm{int}}=-\ln \left\langle\exp \left(-i \int_{0}^{\beta} d \tau e V(\tau, \theta(\tau))\right)\right\rangle_{V} .
$$

Provided the fluctuating field $V$ is sufficiently well described within the Gaussian approximation, we obtain

$$
S_{\mathrm{int}}=\frac{e^{2}}{2} \int_{0}^{\beta} d \tau \int_{0}^{\beta} d \tau^{\prime}\left\langle V(\tau, \theta(\tau)) V\left(\tau^{\prime}, \theta\left(\tau^{\prime}\right)\right)\right\rangle .
$$

The correlator $\langle V V\rangle$ can be expressed via the dielectric susceptibility of the environment $\epsilon(\omega, k)$ as:

$$
\langle V V\rangle=T \sum_{\omega_{n}} \int \frac{d^{3} k}{(2 \pi)^{3}} \frac{4 \pi}{k^{2} \epsilon\left(i\left|\omega_{n}\right|, k\right)} e^{-i \omega\left(\tau-\tau^{\prime}\right)+i \boldsymbol{k} \boldsymbol{X}},
$$

where $\omega_{n}=2 \pi n T$ and $\boldsymbol{X}=\boldsymbol{R}(\tau)-\boldsymbol{R}\left(\tau^{\prime}\right)$. In what follows we will model the environment by a $3 \mathrm{~d}$ diffusive electron gas with $1 / \epsilon(\omega, k) \approx\left(-i \omega+D k^{2}\right) /(4 \pi \sigma)$, where $\sigma$ is the Drude conductivity of this gas and $D=v_{F} l / 3$ is the electron diffusion coefficient. Substituting $1 / \epsilon(i|\omega|, k)$ into (5) and performing integrations we observe that the term $\sim D k^{2}$ yields only an $X$-independent energy shift. Integrating the remaining contribution $\sim\left|\omega_{n}\right|$ over $k \lesssim 1 / l$ in (5) we obtain the result $\propto \min (1 / X, 1 / l) \approx$ $1 / \sqrt{X^{2}+l^{2}}$. Defining $X=2 R \sin \left[\left(\theta(\tau)-\theta\left(\tau^{\prime}\right)\right) / 2\right]$ and summing over $\omega_{n}$, from (4) and (5) we get

$$
\begin{array}{r}
S_{\mathrm{int}}[\theta]=\alpha \int_{0}^{\beta} d \tau \int_{0}^{\beta} d \tau^{\prime} \frac{\pi^{2} T^{2} K\left(\theta(\tau)-\theta\left(\tau^{\prime}\right)\right)}{\sin ^{2}\left[\pi T\left(\tau-\tau^{\prime}\right)\right]}, \\
K(z)=1-\frac{1}{\sqrt{4 r^{2} \sin ^{2}(z / 2)+1}},
\end{array}
$$

where $\alpha=3 /\left(8 k_{F}^{2} l^{2}\right)$ and $r=R / l$. The integral in eq. (6) is understood as a principal value. The divergence at $\tau=\tau^{\prime}$ is then regularized in a standard manner by requiring $K(0)=0$ which explains the origin of the first term in (7). Below we will set $1 / k_{F} \ll l \ll R$ implying that interaction is effectively weak $\alpha \ll 1$ while the parameter $r=R / l$ is large $r \gg 1$.

Perturbation theory. In the non-interacting limit $S_{\mathrm{int}} \rightarrow 0$ the partition function (1) is trivially evaluated, and for the flux-dependent part of the ground state energy at $-0.5<\phi_{x} \leq 0.5$ one finds $E_{0}\left(\phi_{x}\right)=E_{C} \phi_{x}^{2}$.

Let us now take interaction into account. We first assume that interaction effects are weak, in which case it suffices to expand the partition function (11) to the first order in $S_{\text {int }}$ (6). Here it will be convenient for us to rewrite the function $K$ in terms of the Fourier series [6]

$$
K=\sum_{n} a_{n} \sin ^{2}\left[\frac{n\left(\theta(\tau)-\theta\left(\tau^{\prime}\right)\right)}{2}\right]
$$

where $a_{n} \sim(2 / \pi r) \ln (r / n)$ for $1 \leq n \lesssim r$ and $a_{n} \approx 0$ otherwise. After that the calculation reduces to a simple Gaussian integration over the $\theta$-variable in each term of the series. Performing this integration and summing over $n$ one obtains $E_{0}\left(\phi_{x}\right)$ and finds a diamagnetic current $I=(e / 2 \pi)\left(d E_{0} / d \phi_{x}\right)$ with the result

$$
I=\frac{e E_{C}}{\pi}\left[\phi_{x}-\frac{\alpha}{2} \sum_{n=1}^{r} n a_{n} \ln \left(\frac{n+2 \phi_{x}}{n-2 \phi_{x}}\right)\right],
$$

where $-0.5<\phi_{x} \leq 0.5$. The last term in (9) represents the first order correction to $\mathrm{PC}$ in the ring due to Coulomb interaction at $T=0$. This correction is negative, i.e. for the problem in question interactions suppress $\mathrm{PC}$ even in the ground state. For $\phi_{x} \ll 1$ the interaction term in (9) is linear in $\phi_{x}$ and it is small in the parameter $\alpha \sum_{n=1}^{r} a_{n} \sim \alpha \ll 1$ as compared to the non-interacting PC. A somewhat stronger effect of interactions $\sim \alpha \ln r$ was found in ref. [6] within the renormalization group (RG) analysis. Below we will show that already for small $\alpha \ll 1$ suppression of quantum coherence is actually much stronger than it could be expected both from eq. (9) and the RG approach [6].

Nonperturbative effects. Let us first consider the regime of not very low temperatures. In this case the partition function (11) can be evaluated semiclassically. As in [10,11] one can find the classical paths providing the minimum of the total action $S=S_{0}+S_{\text {int }}$ for any winding number $m$. These are the so-called "straight line" paths $\theta_{\mathrm{cl}}(\tau)=2 \pi m T \tau$, describing rotations of the particle around the ring. Substituting the paths $\theta_{\mathrm{cl}}(\tau)$ into the sum (1) one gets

$$
\mathcal{Z} \sim \sum_{m=-\infty}^{\infty} \exp \left(i 2 \pi m \phi_{x}-\frac{\pi^{2} m^{2} T}{E_{C}}-4 \pi|m| \alpha r\right)
$$

In the limit $T \gg E_{C} / \pi^{2}$ it suffices to keep only the terms with $m=0, \pm 1$ and the flux-dependent part of the free energy $F=-T \ln \mathcal{Z}$ can easily be evaluated. Then for PC $I(T)=(e / 2 \pi)\left(d F / d \phi_{x}\right)$ one obtains

$$
I=2 e T \exp \left(-\frac{\pi^{2} T}{E_{C}}-4 \pi \alpha r\right) \sin \left(2 \pi \phi_{x}\right) .
$$

We observe that in addition to trivial reduction of $\mathrm{PC}$ due to thermal fluctuations there is also a $T$-independent term which yields exponential suppression of $I$ provided the perimeter of the ring $2 \pi R$ exceeds the value

$$
L_{\varphi} \sim l / \alpha \sim l\left(k_{F} l\right)^{2} .
$$

Eq. (12) defines the dephasing length for the problem in question. This length does not depend on temperature and is controlled by the effective interaction strength $\alpha$.

The above semiclassical analysis is justified at sufficiently high temperatures, whereas at lower $T \lesssim E_{C}$ 
fluctuations around the classical paths $\theta_{\mathrm{cl}}(\tau)$ become important and our treatment needs to be modified. Provided the suppression of $\mathrm{PC}$ is sufficiently strong one can employ the instanton technique 9, 10].

Nontrivial saddle points $\tilde{\theta}(\tau)$ which describe quantum tunneling of the variable $\theta$ between different topological sectors, e.g. between the states $\theta=0$ and $\theta=2 \pi$, are defined as solutions of the equation $\delta S / \delta \tilde{\theta}=0$. For the action (6), (7) this equation is approximately satisfied for a wide class of sufficiently smooth functions obeying the boundary conditions $\tilde{\theta}(0)=0$ and $\tilde{\theta}(\beta)=2 \pi$. Defining the typical instanton frequency $\Omega$, one finds [9,10] that for $\Omega \lesssim E_{C}$ and $T \ll E_{C}$ the total action $S[\tilde{\theta}(\tau)]$ does not depend on $E_{C}$ and is equal to $S_{\text {int }}[\tilde{\theta}(\tau)]=4 \pi \alpha r$, while for $\Omega>E_{C}$ the instanton contribution gets suppressed by the kinetic energy term $S_{0}$. For $S_{\text {int }}[\tilde{\theta}(\tau)] \gg 1$ both the tunneling amplitude between the states $\theta=2 \pi m$ and $\theta=2 \pi(m \pm 1)$ and the fluxdependent part of the free energy $F$ are exponentially reduced as $\propto \exp (-S[\tilde{\theta}(\tau)])$. Hence, in the limit of small $\phi_{x} \ll 1$ we obtain $I=e E_{C}^{*} \phi_{x} / \pi$, where

$$
E_{C}^{*} / E_{C}=A(T) \exp (-4 \pi \alpha r) .
$$

This result is justified provided $4 \pi \alpha r \gg 1$ and the ratio $E_{C}^{*} / E_{C}$ is sufficiently small. It demonstrates that also at low $T \ll E_{C} \mathrm{PC}$ is strongly suppressed by Coulomb interaction provided the ring perimeter $2 \pi R$ exceeds the dephasing length $L_{\varphi}$ (12). Eq. (13) suggests that at low $T$ interaction-induced decoherence in our model is controlled by the parameter $\alpha r \sim \alpha \sum_{n=1}^{r} n a_{n}$ rather than by $\alpha$ or $\alpha \ln r$. Hence, for $r \gg 1$ the decoherence effect of Coulomb interactions is much stronger than it could be expected both from perturbative and RG approaches.

While the above instanton analysis is applicable for $4 \pi \alpha r \gg 1$, it can hardly provide a quantitative description of PC for moderate $\alpha r$. Furthermore, even for large rings rigorous analytic evaluation of the pre-exponent $A(T)$ in eq. (13) is difficult because the action (6), (7) is strongly non-Gaussian and the standard techniques are hard to employ. On a qualitative level one expects that many non-Gaussian quasi-zero modes should yield a large pre-exponent $A(T)$ increasing upon decreasing $T$.

Quantum Monte Carlo analysis. In order to quantitatively describe reduction of PC at arbitrary values of $\alpha r$ we carried out extensive Monte Carlo (MC) simulations and directly computed $E_{C}^{*}$ as a function of $\alpha$ and $r$. Our method is described in details in ref. [11. In order to discretize the action defined by eqs. (2), (6) and (7) we introduce the Trotter number $N=\beta E_{C}$. We then evaluate path integrals in eq. (11) for different $m$ and determine $E_{C}^{*}(T)=2 \pi^{2} T\left\langle m^{2}\right\rangle_{\phi_{x}=0}$. By increasing $N$ we decrease effective temperature in our simulations. The value $E_{C}^{*}(T)$ grows with decreasing $T$ and finally reaches a plateau which defines the zero temperature value $E_{C}^{*}(\alpha, r)$, see the inset in Fig. 1.

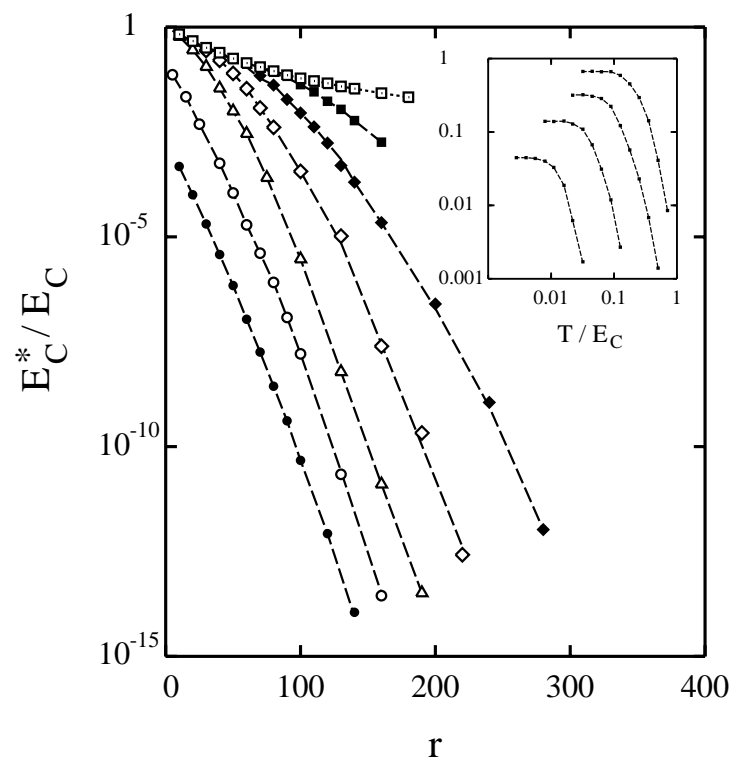

Fig. 1 The value $E_{C}^{*} / E_{C}$ as a function of $r$ for $\alpha=0.019$ and different $T$. From bottom to top: $T / E_{C}=1,0.5,0.14$, 0.06, 0.03 and 0 . The inset: $E_{C}^{*} / E_{C}$ as a function of $T$. From top to bottom: $r=10,30,60$, and 120 .

At relatively high temperatures $T \gtrsim \alpha r E_{C}$ our MC data confirm the semiclassical result (11). Also at lower $T$ we observe dramatic suppression of $E_{C}^{*}$ with increasing $r$, see Fig. 1. At sufficiently large $r$ this suppression turns exponential and at $E_{C} /(4 \pi \alpha r) \lesssim T \lesssim E_{C}$ is well described by eq. (13) with $A(T) \approx \exp \left(c E_{C} / T\right)$ and $c \sim 1$. Thus, eq. (13) holds even at temperatures well below the level spacing $\left(\sim E_{C}\right)$ of the noninteracting problem.

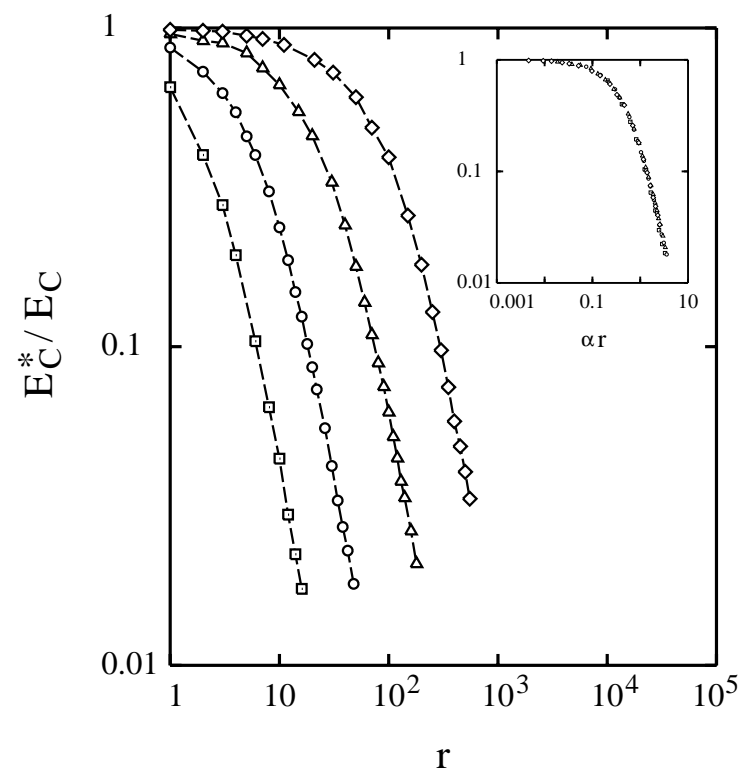

Fig. $2 E_{C}^{*} / E_{C}$ as a function of $r$ at $T=0$ (bottom to top: $\alpha=0.21,0.07,0.019$, and 0.005). The inset: the same data for different $\alpha$ collapse onto one curve if plotted versus $\alpha r$. 
The data corresponding to the exact limit $T=0$ are displayed in Fig. 2 for different $\alpha$. At relatively small $r$ we observe very weak suppression of $E_{C}^{*}$ in agreement with our perturbative result (9). However, at $\alpha r \gtrsim 1$ the ratio $E_{C}^{*} / E_{C}$ rapidly decreases as $E_{C}^{*} / E_{C} \propto r^{-\gamma}$ with $\gamma \approx 1$.8. In order to judge whether at $T=0$ such power law behavior holds at all $r \gtrsim 1 / \alpha$ or it is merely an intermediate regime with further crossover to the exponential dependence (13) (cf. Fig. 1) it is necessary to extend our simulations at large $N$ to values $E_{C}^{*} / E_{C}$ below 0.01 . Such details are, however, of no significance for our conclusions. Much more importantly, our MC data clearly demonstrate drastic suppression of PC by Coulomb interactions even exactly at $T=0$ in rings with $2 \pi R>L_{\varphi}$ as well as the failure of perturbation theory for such values of $R$. Hence, $L_{\varphi}$ (12) remains the relevant scale down to $T=0$, see the inset in Fig. 2 .

Real time calculation. In order to illustrate a relation between the above calculation and real time quasiclassical analysis let us evaluate the return probability for a pair of classical paths which encircle the ring and return to the initial point after time $t$. As in [7] we distinguish two probabilities $W_{1}$ and $W_{2}$ corresponding respectively to two identical and two time-reversed paths. The quantity $W_{1}$ is the standard return probability and $W_{2}$ describes quantum interference between time-reversed paths. The latter quantity is sensitive to quantum coherence and it vanishes in the classical limit.

Without interactions the time reversal symmetry yields $W_{1}=W_{2}=W^{(0)}$. The effect of interactions is accounted for by the influence functional [13] $\mathcal{F}=$ $\exp \left(i S_{R}-S_{I}\right)$, and the probabilities are now equal to $W^{(0)} \mathcal{F}$. For our model the actions $S_{R}$ and $S_{I}$ are evaluated by integrating out the fluctuating electromagnetic field produced by the electron gas. Similarly to eqs. (4), (5) both $S_{R}$ and $S_{I}$ are expressed in terms of the inverse dielectric function $1 / \epsilon(\omega, k)$ of the electron environment.

For two identical paths one finds $S_{R}=S_{I}=0$, i.e. the probability $W_{1}=W^{(0)}$ is not affected by interactions at all. For a pair of time-reversed paths one also has $S_{R}=0$, but the action $S_{I}$ is now positive $S_{I}>0$ and, hence, $W_{2}=W^{(0)} \exp \left(-S_{I}\right)$. In order to evaluate $S_{I}$ it is convenient to "unfold" the time-reversed path into two straight lines $\boldsymbol{r}_{1}\left(t_{1}\right)=\boldsymbol{v} t_{1}$ and $\boldsymbol{r}_{2}\left(t_{1}\right)=\boldsymbol{v}\left(t-t_{1}\right)$, where $0 \leq t_{1} \leq t$. Inserting these paths into $S_{I}$ [2] we get

$$
\begin{aligned}
S_{I}= & \frac{e^{2}}{2} \int \frac{d \omega}{2 \pi} \int \frac{d^{3} k}{(2 \pi)^{3}} \operatorname{Im}\left(\frac{-4 \pi}{k^{2} \epsilon(\omega, k)}\right) \operatorname{coth} \frac{\omega}{2 T} \\
& \times \int_{0}^{t} d t_{1} \int_{0}^{t} d t_{2} \mathrm{e}^{-i \omega\left(t_{1}-t_{2}\right)} \mathcal{I}\left(t_{1}, t_{2}\right),
\end{aligned}
$$

where $\mathcal{I}=2 \cos \left(\boldsymbol{k} \boldsymbol{v} t_{-}\right)-2 \cos \left[\boldsymbol{k} \boldsymbol{v}\left(t-t_{+}\right)\right]$and $t_{ \pm}=t_{1} \pm t_{2}$. In the long time limit one finds

$$
S_{I}=\frac{e^{2} t}{2 \pi v} \int_{0}^{1 / l} d k \int_{-k v}^{k v} d \omega \frac{\omega \operatorname{coth} \frac{\omega}{2 T}}{4 \pi \sigma k} .
$$

Defining the path length $L=v t$, at $T=0$ we obtain

$$
S_{I}=\frac{e^{2} L}{16 \pi^{2} \sigma l^{2}} \sim \frac{L}{l \alpha} .
$$

Hence, similarly to $\mathrm{PC}$, at $T \rightarrow 0$ the probability $W_{2}$ is suppressed by interactions beyond the length (12) and the ratio $W_{2} / W_{1} \propto \exp \left(-L / L_{\varphi}\right)$ vanishes at large $L$. The latter result proves that reduction of $\mathrm{PC}$ is due to zero temperature decoherence and is not just a renormalization effect. The same conclusion follows from studying the $\mathrm{AB}$ conductance which we do not present here.

Finally, let us define the dephasing time $\tau_{\varphi}$. For ballistic particle motion considered here we set $\tau_{\varphi}=L_{\varphi} / v$. In particular, for $v \sim v_{F}$ we get $\tau_{\varphi} \sim \tau_{e}\left(k_{F} l\right)^{2}$, where $\tau_{e}=l / v_{F}$ is the elastic scattering time for electrons. The latter expression for $\tau_{\varphi}$ is identical to the electron dephasing time obtained in ref. [2] for $3 \mathrm{~d}$ disordered conductors. This observation is consistent with the idea [14] that the results of weak localization and persistent current experiments may be closely related. In particular, we expect both to yield the same dephasing length $L_{\varphi}$ at low $T$.

We are grateful to F. Guinea, M. Paalanen and G. Schön for interesting discussions.

[1] P. Mohanty, E.M.Q. Jariwala, and R.A. Webb, Phys. Rev. Lett. 78, 3366 (1997).

[2] D.S. Golubev and A.D. Zaikin, Phys. Rev. Lett. 81, 1074 (1998); Phys. Rev. B 59, 9195 (1999).

[3] D.S. Golubev, A.D. Zaikin, and G. Schön, J. Low. Temp. Phys. 126, 1355 (2002); I.L. Aleiner, B.L. Altshuler, and M.G. Vavilov, ibid. 126, 1377 (2002); D.S. Golubev and A.D. Zaikin, in preparation.

[4] P. Cedraschi, V.V. Ponomarenko, and M. Büttiker, Phys. Rev. Lett. 84, 346 (2000); Ann. Phys. 289, 1 (2001).

[5] U. Gavish, Y. Levinson, and Y. Imry, Phys. Rev. B 62, 10637 (2000); Y. Imry, cond-mat/0202044.

[6] F. Guinea, Phys. Rev. B 65, 205317 (2002).

[7] D.S. Golubev and A.D. Zaikin, Physica B 255, 164 (1998).

[8] F. Guinea and G. Schön, Europhys. Lett. 1, 585 (1986); G. Falci, G. Schön, and G.T. Zimanyi, Phys. Rev. Lett. 74, 3257 (1995); W. Hofstetter and W. Zwerger, ibid. 78, 3737 (1997); J. König and H. Schoeller, ibid. 81, 3511 (1998).

[9] S.V. Panyukov and A.D. Zaikin, Phys. Rev. Lett. 67, 3168 (1991); J. Low Temp. Phys. 73, 1 (1988).

[10] X. Wang and H. Grabert, Phys. Rev. B 53, 12621 (1996).

[11] C.P. Herrero, G. Schön, and A.D. Zaikin, Phys. Rev. B 59, 5728 (1999) and further references therein.

[12] F. Marquardt and C. Bruder, Phys. Rev. B 65, 125315 (2002).

[13] See, e.g., R.P. Feynman and A.R. Hibbs, Quantum Mechanics and Path Integrals (McGraw Hill, NY, 1965).

[14] P. Mohanty, Ann. Physik (Leipzig) 8, 549 (1999). 